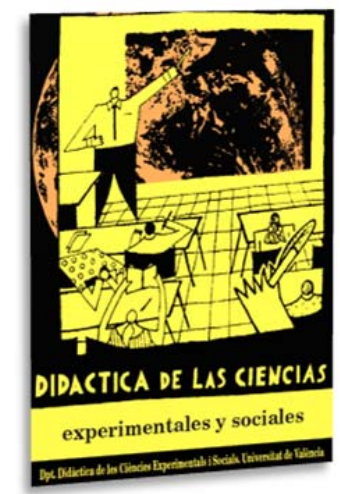

\title{
¿Cómo evaluar la reflexión sobre la práctica docente? Un ejemplo en la formación inicial del profesorado de Biología y Geología
}

\author{
How to evaluate reflection on teaching \\ practice? An example in the initial training of \\ Biology and Geology teachers
}

DOI: $10.7203 / D C E S .37 .14797$

\author{
María José Sáez Bondía \\ Universidad de Zaragoza, msaezbo@unizar.es \\ ORCID iD: https://orcid.org/0000-0002-8733-1501 \\ Ángel Luis Cortés Gracia \\ Universidad de Zaragoza, acortes@unizar.es \\ ORCID iD: https://orcid.org/0000-0002-7075-9683
}

\begin{abstract}
RESUMEN: La palabra reflexión aparece en numerosas ocasiones cuando se hace referencia a la formación inicial del profesorado. No obstante, son pocas las ocasiones en las que se define qué se entiende por reflexión y, aún menos, cómo evaluarla de un modo sistemático. Este trabajo, en el contexto de la formación inicial del profesorado de educación secundaria, muestra una estrategia para evaluar la reflexión de estudiantes del máster de profesorado de Biología y Geología sobre una actividad relacionada con las salidas a entornos próximos. Para ello, se realiza el análisis de las declaraciones de los estudiantes sobre las implicaciones didácticas de la secuencia realizada. Los resultados muestran que solo alcanzan niveles medioaltos de reflexión un escaso porcentaje de las declaraciones. Se proponen criterios para categorizar el nivel de reflexión sobre situaciones de aula y se discuten las posibilidades de la metodología de análisis empleada.
\end{abstract}

PALABRAS CLAVE: reflexión, evaluación, formación inicial del profesorado, metodología de investigación, enseñanza de las ciencias.

ABSTRACT: Reflection in initial teacher training is present in several contexts. Nevertheless, there are few occasions in which reflection is defined and even less in which it is systematically evaluated. This paper, in the context of initial teacher training for secondary education, shows a strategy used to evaluate the reflection on a fieldwork activity by a group of Biology and Geology pre-service teachers. To this end, an analysis is made of the students' statements on the didactic implications of the teaching sequence carried out. The results show that only a small percentage of the statements reach medium-high levels of reflection. Criteria are proposed to categorize the level of reflection on classroom situations and the possibilities of the analysis methodology used are discussed.

KEYWORDS: reflection, evaluation, initial teacher training, research methodology, science education.

Fecha de recepción: abril de 2019 Fecha de aceptación: noviembre de 2019

El presente trabajo se enmarca en el proyecto de investigación EDU2016-76743-P, financiado por el MINECO. Grupo de referencia BEAGLE Investigación en Didáctica de las Ciencias Naturales (Gobierno de Aragón y Fondo Social Europeo) e Instituto de Investigación en Ciencias Ambientales de Aragón (IUCA/UNIZAR). 


\section{INTRODUCCIÓN}

La razón que articula este trabajo parte del deseo de fomentar en el profesorado en formación una actitud crítica y reflexiva sobre la práctica docente. Este deseo impregna las memorias y guías docentes de las titulaciones de los Grados en Maestro de Educación Infantil y Primaria y del Máster de Profesorado. Reflexionar como objetivo formativo no es exclusivo del centro y la universidad donde se ha desarrollado esta investigación, sino que coincide con lo establecido en los Libros Blancos que precedieron la implantación de las titulaciones (Agencia Nacional de Evaluación de la Calidad y Acreditación [ANECA], 2005) y que se ha recogido en diversos manuales prácticos así como en las guías docentes de distintas universidades hasta la actualidad (Imbernón, 2017; Lledó y Giner, 2009; Pérez Gómez, 2010).

Sin embargo, pocas veces estos documentos indican a qué se refieren sus autores con "reflexión" y, menos aún, cómo conseguirlo o evaluarlo. Esta problemática relacionada con el término "reflexión" y con la necesidad de fomentar la misma no es algo nuevo. Goodman (1987) ya concretaba que, tal y como sucede con otros términos en la enseñanza, "la reflexión parece haber adquirido cierto carácter de eslogan, hasta el punto de significar cosas muy distintas para las diferentes personas" (p.239) y enfatizaba en la necesidad de que los profesores en formación inicial analizasen las relaciones existentes entre la teoría y la práctica docente, aspecto que en la actualidad sigue preocupando desde diferentes áreas de conocimiento (Fernández-Fernández et al., 2016; Russel, 2012).

Ante esta situación, la primera pregunta que surgió fue la siguiente: ¿es posible crear contextos teórico-prácticos durante la formación del profesorado en los que los estudiantes tengan la oportunidad de reflexionar acerca de la práctica docente? Obviamente, los periodos de prácticas en los centros escolares representarían el momento ideal, pero de acuerdo a lo establecido en las memorias y las guías, ese proceso reflexivo se debería fomentar en la mayoría de las asignaturas. Por lo tanto, debíamos encontrar un escenario en el que poner a prueba a los futuros docentes, demandando esa reflexión sobre las necesidades, expectativas y posibilidades de aplicación de una propuesta concreta (Russel, 2012). Y ahí no acaba todo, ya que en caso de lograr que estos estudiantes reflexionen sobre la práctica docente, ¿cómo podemos saber si realmente lo están haciendo?

Este trabajo muestra la estrategia de análisis llevada a cabo para evaluar la reflexión a partir de una sesión de discusión en un grupo de estudiantes del Master de Profesorado de Secundaria de la Universidad de Zaragoza (especialidad de Biología y Geología) al sintetizar una secuencia de actividades prácticas contextualizadas en las salidas de campo con un doble objetivo:

- Conocer el efecto "reflexivo" de la puesta en práctica de la secuencia de actividades llevada a cabo en la formación inicial del profesorado de Biología y Geología.

- Establecer una estrategia de evaluación sistemática de la reflexión a partir del discurso que considere los saberes propios de la temática tratada.

\section{MARCO TEÓRICO}

\subsection{Reflexión en la formación inicial del profesorado}

Reflexión, pensamiento reflexivo y desarrollo de profesionales reflexivos son palabras que aparecen habitualmente en los programas de formación docente con muy diversas interpretaciones (Goodman, 1987; Russel, 2012). Así, un elemento común en los distintos enfoques dados al concepto reflexión es que se asocia a un problema que deriva en una situación de sorpresa o incertidumbre (Hatton y Smith, 1995; Loughran, 2002). 
Las obras de Schön acerca de la formación de profesionales reflexivos (1992) o cómo piensan los profesionales como actúan (1998) son grandes referentes cuando se aborda el concepto de reflexión del profesorado en formación y en ejercicio. Así, Schön, retomando el trabajo de Dewey (1938) sobre la teoría de la indagación, propone un cambio de paradigma en la formación de profesionales dado el distanciamiento entre la "teoría" dada en las clases universitarias y la realidad profesional. Propone como solución un acercamiento de la práctica a las aulas universitarias. Es decir, el aprendizaje a través de la experiencia profesional y la reflexión en, desde y sobre la acción de dicha experiencia. Diversos trabajos centrados en las competencias profesionales también introducen la reflexión como elemento fundamental, si bien se suelen referir al desarrollo de estas competencias en contextos de prácticas externas en el medio socio-profesional (Peinado y Abril, 2016).

La reflexión desde el enfoque aportado por Schön (1998) se refiere al trabajo real en un aula. Sin embargo, cuando aludimos a profesores en formación inicial, quizás el modo en el que este autor enfoca la reflexión (o la contextualiza) no sería el más adecuado a no ser que estuviésemos hablando de su reflexión en el momento en el que realizan las prácticas en los centros escolares. A pesar de ello, muchas ideas de las aportadas por Schön pueden ser transferibles al contexto en el que nos movemos y así lo hacen autores como Perrenoud (2004).

Siguiendo la línea de Schön (1998), retomamos sus antecedentes para aclarar el concepto polisémico de reflexión volviendo a Dewey" (1989) y su obra "Cómo pensamos". En ella, esboza detalladamente qué entiende por pensamiento reflexivo, da motivos por los que orientar hacia este tipo de pensamiento como forma de pensar adecuada para aprender, especifica sus fases y concreta aquellos elementos que podrían servir para orientar ese pensamiento.

Podemos decir que la idea de reflexión expuesta por Dewey incluye cuatro criterios fundamentales que concreta Rodgers (2002) muchos años después: 1) se trata de un proceso de creación de significados que mueve al aprendiz de una experiencia a la siguiente con una mayor comprensión de sus relaciones y conexiones con otras ideas y experiencias; 2) es un modo de pensar sistemático, riguroso y disciplinado con sus raíces en la investigación científica; 3) tiene que darse en comunidad y 4) requiere actitudes que valoran el crecimiento personal e intelectual de uno mismo y de los demás.

Por tanto, la reflexión puede extenderse al cuestionamiento de una situación de aula, del enfoque dado a los contenidos trabajados, de los recursos empleados y de la evolución previsible de una determinada situación (Perrenoud, 2004). Es más, dicha reflexión puede darse a partir de un problema vinculado a un fenómeno, acontecimiento u objeto cualquiera. Por ejemplo, la problemática que puede surgir entre diferenciar una hoja simple de una compuesta en un determinado árbol puede iniciar un proceso reflexivo que necesita de una comprobación. Así, podríamos hablar en este caso de una reflexión desde el contenido como medio para el aprendizaje (Dewey, 1989). Sin embargo, lograr solo reflexión (en mayor o menor medida) acerca del contenido no es suficiente en la formación del profesorado. Es necesario fomentar una reflexión sobre los aspectos didácticos de la temática trabajada. Es decir, fomentar la reflexión, llamémosla "didáctica", para el desarrollo del Conocimiento Didáctico del Contenido en estos estudiantes (Medina y Jarauta, 2013)

A partir de estas ideas iniciales han sido añadidas diversas estrategias específicas con objeto de fomentar un pensamiento reflexivo. Por ejemplo, el uso de entrevistas personales (Smith, 1991) $\mathrm{y}$ de textos escritos en diferentes formatos (Hatton y Smith, 1995; Mena, García Rodríguez y Tillema, 2012; Zeichner, 1987), el auto-vídeo análisis de la actuación de los profesores en formación inicial en el aula (Simon y Campbell, 2012) o las discusiones grupales sobre aspectos vinculados a la enseñanza (Yoon y Kim, 2010). Estas estrategias permiten que los estudiantes hagan explícitos sus pensamientos y acciones, favoreciendo así la reflexión (Hatton y Smith, 1995).

\footnotetext{
${ }^{1}$ La versión original de esta obra data de 1933.
} 
En la secuencia de actividades utilizadas en este trabajo utilizamos como motor de reflexión "didáctica" la visualización de las grabaciones de vídeo y su posterior análisis a través del texto escrito y el uso de discusiones grupales. Esta estrategia ha sido empleada ampliamente tanto en profesores en formación inicial como en ejercicio para fomentar la reflexión (Gaudin y Charliès, 2015). De hecho, desde la perspectiva de Dewey, autores como Lussi Borer, Ria, Durand y Muller (2014) identifican dos formas de razonamiento cuando los profesores en formación inicial visualizan la actuación de otros diferentes a ellos. Inicialmente, observan situaciones y puede haber también una descripción, interpretación, explicación e incluso evaluación de lo que identifican. En segundo lugar, pueden comparar las situaciones que han visualizado con experiencias propias.

Son diversas las estrategias en las que se emplea la visualización de grabaciones de vídeo (Amobi, 2005; Gaudin y Charliès, 2015) y que han demostrado incrementar la reflexión de los profesores en formación y en ejercicio. Dicha reflexión se ve favorecida con el uso de herramientas que permiten focalizar el análisis ya que, de este modo, se ofrece la oportunidad a los profesores en formación de visualizar, analizar y perfeccionar su práctica docente antes, durante y después de su trabajo en el aula (Rich y Hannafin, 2009).

A pesar de la cantidad de estudios centrados en la reflexión son pocos los que intentan registrarla de algún modo. De entre los pocos trabajos que se preocupan por evidenciar el pensamiento reflexivo y, por ende, la reflexión, cabe destacar el de Hatton y Smith (1995). Estos autores, a partir de la categorización establecida por Van Manen (1977) y las ideas de Schön (1992 y $1998^{2}$ ), establecen, analizando textos escritos, 4 niveles de reflexión de creciente complejidad: 1) escritura descriptiva, cuando se describe de una situación (no se considera reflexión), 2) reflexión descriptiva, cuando se dan razones sobre una situación; 3) reflexión dialógica, cuando se dan razones justificadas sobre una situación y 4) reflexión crítica, cuando las justificaciones de una situación van más allá considerando aspectos políticos o sociales, entre otros. Dicha categorización también ha sido empleada para el análisis del nivel de reflexión en textos escritos por autores como Mena et al. (2012).

Otros autores como Toom, Husu y Patriakainen (2014) retoman las ideas de Dewey (1989) para intentar conocer los procesos de reflexión de los estudiantes de magisterio en prácticas a través de portafolios. Para ello, consideran 5 categorías derivadas de las fases o momentos de la acción reflexiva definidas por éste: 1) introspección, cuando se retoman los pensamientos sobre algo vinculado a la enseñanza; 2) asociación, cuando esos hechos se vinculan con conocimientos anteriores; 3) integración, cuando relaciona su experiencia con un marco teórico; 4) validación, cuando establece un balance entre sus pensamientos y sus acciones y 5) apropiación, entendida como el momento en el que declara hacerse dueño de sus aprendizajes.

Como ya hemos indicado anteriormente, el pensamiento reflexivo constituye un modo determinado de pensar, mientras que la acción reflexiva hace referencia a llevar a cabo acciones diversas para dar soluciones a problemas (Dewey, 1989). Ambos procesos están íntimamente relacionados (Schön, 1998). Así, la reflexión es, desde nuestro punto de vista, un proceso derivado de un pensamiento que es sistemático, viene determinado por unas fases y constituye un medio para el aprendizaje, ya que permite la integración de una serie de saberes. De este modo, la visualización de vídeos y su posterior análisis y discusión es una herramienta que puede fomentar el pensamiento reflexivo y la reflexión al mismo tiempo que permite detectar indicios para reconocerlos.

\subsection{Conocimiento Didáctico del Contenido y reflexión}

La formación de los futuros profesores de ciencias se enmarca bajo una serie de saberes incluidos dentro del Conocimiento Didáctico del Contenido de sus correspondientes disciplinas científicas (en adelante, CDC) (Shulman, 1986; Acevedo, 2009). El CDC incluye "las formas más regulares de enseñar una asignatura, de representar esas ideas, las más poderosas analogías,

\footnotetext{
${ }^{2}$ Las versiones originales de estos trabajos empleadas por Hatton y Smith datan de 1983 y 1987 respectivamente.
} 
ilustraciones, ejemplos, explicaciones, y demostraciones. Los modos en los que representar y formular la materia de modo que la hagan comprensible a otros" y "requiere una comprensión de lo que hace fácil o difícil a una determinada materia a los estudiantes a los que va dirigida" (Shulman, 1986, p. 9).

Esta definición dada por Lee Shulman hace más de tres décadas sigue tomándose como referencia cuando se habla de los saberes necesarios de un profesor o cómo ser un buen profesor (Nóvoa, 2009). No obstante, ha ido sufriendo modificaciones en relación a los elementos que constituyen esos saberes necesarios y su relación entre ellos. Shulman (1987) asociaba la planificación reflexiva del profesor como algo necesario para ser un buen enseñante y la idea de incluir el proceso reflexivo se ha mantenido en posteriores modelos sobre el CDC (Nilsson, 2008; Park y Oliver, 2008). Autores como Marcelo (1993) consideran que la reflexión enmarcada dentro del CDC facilita que los docentes realicen un auto-análisis de sus ideas relativas a lo que enseñan y el modo en el que las enseñan.

Parece que existe un acuerdo en que la reflexión del profesor constituye un nexo entre los diferentes saberes que debería de poseer. No obstante, existen diversos modelos sobre los saberes del profesor que constituyen el CDC y ello ha generado controversia entre los investigadores del área (Borowski et al., 2011). De entre estos modelos conducentes a la caracterización de los saberes destacamos el de Park y Oliver (2008). Tras una profunda revisión sobre el CDC, sus definiciones y saberes incluidos, estos autores elaboraron un modelo que facilita la interpretación de esos saberes necesarios en un profesor. Este sistema de representación fue denominado como Modelo Hexagonal del Conocimiento Didáctico del Contenido.

El Modelo Hexagonal del CDC integra componentes fundamentales distribuidos gráficamente en un hexágono en los que se incluyen, a su vez, sub-componentes que ayudan a definirlos o categorizarlos. En líneas generales, incluye: 1) el conocimiento de los estudiantes por parte del profesor (sus dificultades de aprendizaje para un determinado tema, sus ideas alternativas, entre otros); 2) el conocimiento del currículum (tanto horizontalmente como verticalmente); 3) las orientaciones dadas para enseñar ciencias (incluidas las decisiones tomadas, las creencias sobre los objetivos de la enseñanza de las ciencias o la naturaleza de la ciencia); 4) el conocimiento sobre la evaluación de los aprendizajes de ciencias (estrategias para evaluar o criterios específicos); 5) el conocimiento de estrategias de enseñanza de las ciencias (como estrategias específicas para un tema concreto) y 6) la autoeficacia del profesor (relacionada con la seguridad para tratar un contexto específico o un tema concreto).

Asimismo, estos autores enfatizan que la interrelación entre estos componentes está mediada por un proceso reflexivo. En éste, la adquisición de todos estos saberes implica una relación dinámica en la que la práctica docente y su conocimiento en y sobre la misma tienen un papel importante. Estos últimos términos se asocian a la idea de reflexión en y sobre la acción definidos por Schön (1992), ya que el conocimiento y la forma de adquirir dicho conocimiento se entrelazan dentro de un contexto práctico (Eraut, 1994).

\section{MetodologíA}

\subsection{Contexto de la investigación y participantes}

La actividad realizada con la intención de fomentar la reflexión didáctica fue llevada a cabo en la especialidad de Biología y Geología del Máster de Profesorado de Secundaria de la Universidad de Zaragoza y se relacionaba con las salidas de campo. Ya que en el contexto de la asignatura en el que aplicó la actividad los estudiantes no están inmersos en situaciones de aula real, se propuso la realización de una especie de juego de rol como vía para que los estudiantes reflexionasen sobre las implicaciones didácticas de la realización de una salida de campo en un entorno cercano al centro de enseñanza. 
Previamente a la realización de la actividad, los estudiantes habían recibido información sobre las recomendaciones didácticas relacionadas con las salidas de campo, atendiendo a autores como Orion y Hofstein (1994) o Pedrinaci (2012) y unas nociones muy básicas sobre botánica y sobre lo que se esperaba de la propia actividad. Tras dicha introducción, se propuso a los estudiantes la puesta en marcha de una salida a los jardines del campus universitario con el objetivo de conocer qué especies de árboles y arbustos se podrían encontrar y qué recursos didácticos podían emplear para su identificación. En la actividad participaron 21 estudiantes agrupados en cuatro equipos de trabajo. Uno de los equipos se constituiría como grupo de "expertos". Estaba formado por aquellos estudiantes con más conocimientos sobre botánica (titulados en Biología, Ingeniería Forestal o Ciencias Ambientales), que debían asumir el papel de profesores, desplazándose por el campus para resolver las dudas y orientar al resto de los equipos. Los tres equipos restantes, formados principalmente por titulados en Geología, Bioquímica y Veterinaria, asumirían el papel de estudiantes, debiendo identificar un número determinado de ejemplares y prestando especial atención a las dificultades que se encontraban durante la realización de la actividad práctica. Cada equipo de "estudiantes" disponía de una plantilla-guía donde anotar las características de los ejemplares observados y un libro de identificación de especies adaptado al ámbito escolar (Carrasquer, Álvarez, Lafuente y Pérez, 2001). No obstante, el modo en el que se aportaban los materiales seguía un orden diferente para cada equipo. En concreto, el primer equipo usaba directamente el libro de identificación en el campo para poder identificar "in situ" los ejemplares y no tenía por qué anotar las características en el campo, aunque posteriormente, en el aula, debía incorporar las características principales de cada ejemplar identificado en la plantilla-guía; el segundo equipo anotaba en la plantilla-guía las características de los ejemplares en el campo, sin disponer de otros recursos para la identificación y, posteriormente, debía identificar los ejemplares en el aula con el mismo libro utilizado por el primer grupo; finalmente, el tercer equipo tenía las dos herramientas (plantilla-guía y libro de identificación) disponibles durante toda la actividad.

De esta forma se pretendía que desde los distintos papeles adoptados, los estudiantes pudieran discutir sobre los recursos didácticos y las estrategias de identificación. No obstante, este trabajo no se ha centrado en el análisis de estas variables, aunque parece necesario comentarlas para comprender el contexto de la sesión de discusión.

Todo el proceso fue grabado en vídeo por uno de los participantes de cada equipo y las grabaciones recogidas fueron empleadas con posterioridad para discutir sobre las ventajas e inconvenientes de llevar a cabo la actividad de las diferentes formas propuestas y sobre el papel asumido en cada caso por los "expertos/profesores". Las declaraciones de los participantes fueron plasmadas en diferentes cuestionarios escritos a lo largo de la actividad donde se demandaban respuestas relacionadas con las dificultades que podían encontrar los estudiantes de secundaria y propuestas de mejora de la actividad (Sáez Bondía y Cortés Gracia, 2019).

En una última sesión de la secuencia práctica, todo el grupo-clase puso en común las distintas anotaciones, observaciones o percepciones que, desde el punto de vista didáctico, fueron recogiendo a lo largo de la secuencia. Esta discusión grupal fue grabada también en vídeo y empleada para evaluar el nivel de reflexión sobre la actividad realizada.

\subsection{Análisis de los datos}

El Modelo Hexagonal del CDC propuesto por Park y Oliver (2008) puede tomarse como referencia para estructurar el análisis de los esquemas reflexivos. No obstante, dada la dificultad de interpretación de algunos de los componentes del hexágono considerados desde una perspectiva generalista, en la tabla 1 mostramos aclaraciones con respecto a esos elementos para el contexto en el que se ha desarrollado la investigación.

Con el fin de poner a prueba la metodología de análisis propuesta en este trabajo, como fuente de información se utilizó el conjunto de declaraciones de los estudiantes durante la sesión final de la 
secuencia de actividades a través de un análisis del discurso (Parker, 1992) que recogía comentarios y opiniones sobre el desarrollo completo de toda la secuencia. Asimismo, se tuvieron en consideración tanto las grabaciones de vídeo realizadas durante las tareas de recogida de datos de campo e identificación de ejemplares como las respuestas dadas en los cuestionarios (ver Sáez Bondía y Cortés Gracia, 2019). De este modo, la información recogida a través de estas tres fuentes nos serviría para validar los datos obtenidos: triangulación de datos y validación del respondiente (Simons, 2011).

TABLA 1. Adaptación del Modelo Hexagonal del CDC de Park y Oliver (2008).

\begin{tabular}{|c|c|c|}
\hline $\begin{array}{c}\text { Componentes del } \\
\text { hexágono de Park y Oliver }\end{array}$ & $\begin{array}{l}\text { Nombre asignado para el } \\
\text { contexto estudiado }\end{array}$ & Sub-componentes incluidos \\
\hline $\begin{array}{l}\text { Orientaciones en la } \\
\text { enseñanza de las ciencias }\end{array}$ & $\begin{array}{l}\text { Orientaciones en la } \\
\text { propuesta }\end{array}$ & $\begin{array}{c}\text { Objetivos de aprendizaje } \\
\text { declarados o las decisiones } \\
\text { tomadas por el profesor durante la } \\
\text { actividad. }\end{array}$ \\
\hline $\begin{array}{l}\text { Conocimiento del currículo } \\
\text { de ciencias }\end{array}$ & Materia de estudio & $\begin{array}{l}\text { Materia de estudio como saber y } \\
\text { su relación con el currículo. }\end{array}$ \\
\hline $\begin{array}{l}\text { Conocimiento de los } \\
\text { saberes de los estudiantes }\end{array}$ & Estudiantes & $\begin{array}{c}\text { Dificultades que pueden tener los } \\
\text { estudiantes, sus intereses o } \\
\text { necesidades. }\end{array}$ \\
\hline Autoeficacia del profesor & Seguridad del profesor & $\begin{array}{l}\text { Seguridad del profesor tanto sobre } \\
\text { la materia de estudio como con el } \\
\text { contexto de aula. }\end{array}$ \\
\hline $\begin{array}{l}\text { Conocimiento de } \\
\text { estrategias para la } \\
\text { enseñanza de las ciencias }\end{array}$ & $\begin{array}{l}\text { Estrategias de enseñanza y } \\
\text { aprendizaje }\end{array}$ & $\begin{array}{c}\text { Estrategias de enseñanza y } \\
\text { aprendizaje tales como el diseño } \\
\text { de propuestas, los materiales a } \\
\text { emplear o salidas de campo. Se } \\
\text { incluyen aspectos relativos al rol } \\
\text { del profesor desde una perspectiva } \\
\text { teórica. }\end{array}$ \\
\hline $\begin{array}{c}\text { Conocimiento sobre la } \\
\text { evaluación de la enseñanza } \\
\text { de las ciencias }\end{array}$ & Evaluación & $\begin{array}{l}\text { Aspectos relativos a la evaluación } \\
\text { de los aprendizajes y a las } \\
\text { herramientas de evaluación. }\end{array}$ \\
\hline
\end{tabular}

Fuente: elaboración propia

Inicialmente, la sesión de discusión analizada, grabada en vídeo y transcrita literalmente, se estructuró en tres momentos atendiendo a los temas de interés que surgieron durante la misma y que se muestran en el apartado de resultados. Para cada uno de los temas de interés se utilizó la estructura hexagonal como herramienta sobre la que ir incluyendo las diferentes declaraciones realizadas por los estudiantes en los componentes o sub-componentes del CDC, atendiendo a Ravanal Moreno y López-Cortés (2016). De este modo, las declaraciones fueron sintetizadas y articuladas alrededor de un hexágono en forma de "enunciados" que resumían las declaraciones utilizando nexos entre las ideas principales (gráfico 1). Los enunciados contienen nexos orientativos (sobre flechas) y palabras relevantes (encuadradas) que permiten hacernos una idea de la declaración realizada por un estudiante. Así, tanto los nexos (flechas) como las palabras relevantes (rectángulos) de un enunciado concreto formulado por un estudiante dentro de un tema de interés están representados con los mismos colores sobre el hexágono de referencia.

La información obtenida a partir de la interpretación de los enunciados fue reducida considerando el tipo y número de relaciones establecidas entre componentes del CDC dentro de cada tema de interés. De este modo, no solo podíamos hacernos una idea de los componentes del 
hexágono que se ponían en juego durante la sesión, sino de la relación que establecía el grupo de estudiantes entre éstos. Dichas relaciones nos permitían visibilizar la presencia de reflexión didáctica sobre la actividad a través de las declaraciones de los mismos ya que, tal y como especifican Park y Oliver (2008), la integración de los diferentes elementos del CDC está mediada por un proceso reflexivo.

GRÁFICO 1. Ejemplo de la estructura seguida para el análisis del discurso a partir de los enunciados. Las flechas con el mismo color resumen las ideas expuestas por los estudiantes. Los componentes y subcomponentes del CDC aparecen como elipses y las palabras relevantes como rectángulos.

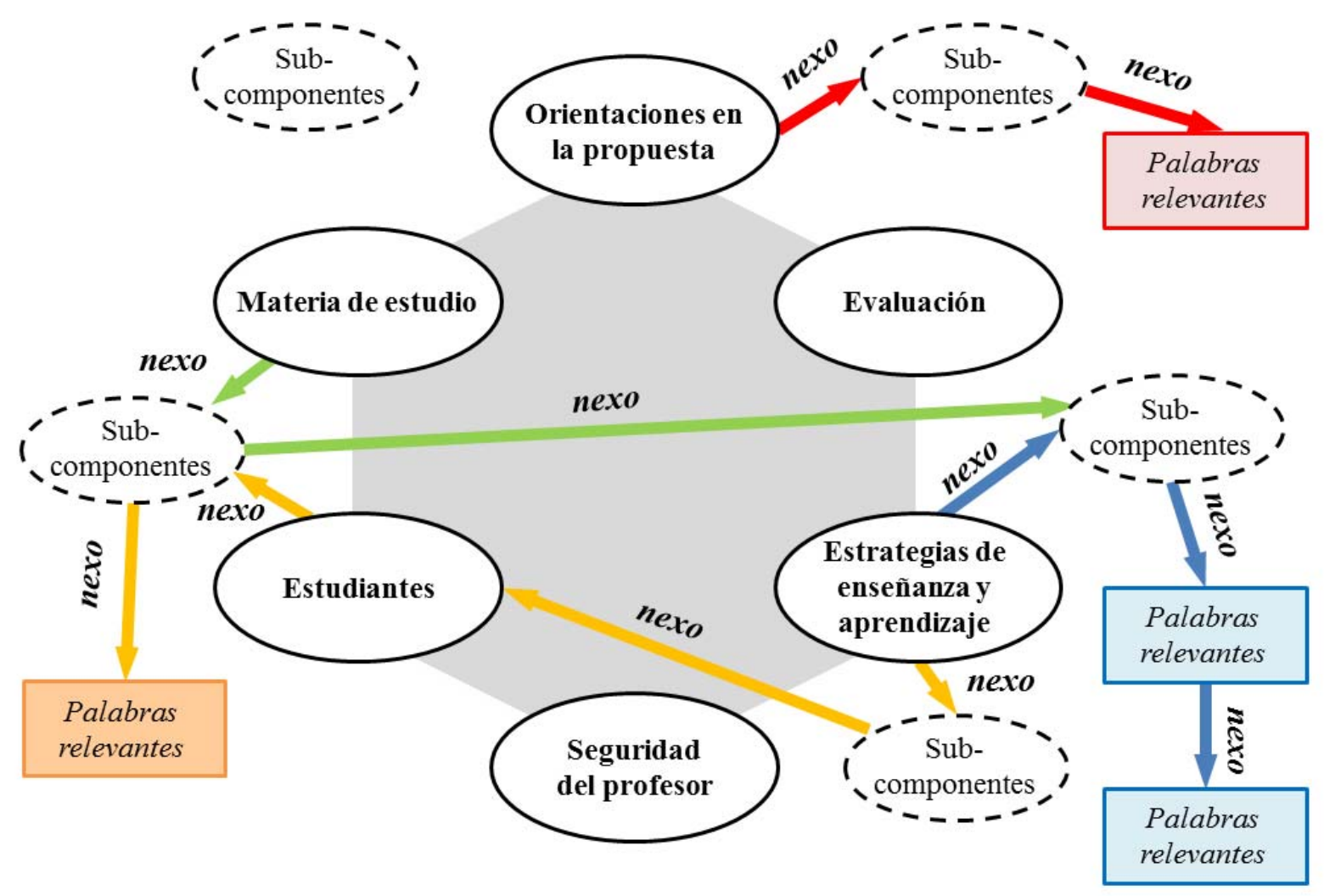

Fuente: elaboración propia

Por otra parte, en el análisis tuvimos en cuenta el tipo de relaciones establecidas entre componentes o dentro del mismo componente con la finalidad de determinar el nivel de reflexión presente en las declaraciones de los estudiantes (tabla 2). Para construir el sistema de categorías que aparece en la tabla 2 se tomó como referencia la categorización de los niveles de reflexión de Hatton y Smith (1995) mostrados en el marco teórico de este trabajo. En el sistema de categorías empleado no se consideró el nivel 4 de reflexión propuesto por estos autores, ya que incluía aspectos sociales y políticos de la educación, no trabajados en el caso de la propuesta planteada. El resto de niveles fueron adaptados al contexto en el que nos movíamos. Desde esa perspectiva, no solo se consideró la existencia de relaciones entre componentes, sino también el tipo de relaciones que se establecían entre ellos, de modo que, cuantos más elementos se relacionan y mayor complejidad "reflexiva" alcanza el enunciado derivado de las declaraciones de los estudiantes, mayor será el nivel de reflexión. 
TABLA 2. Niveles de reflexión asociados a los tipos de relaciones entre componentes del hexágono identificados en los enunciados

\begin{tabular}{ccc}
\hline $\begin{array}{c}\text { Categoría } \\
\text { (nivel reflexivo) }\end{array}$ & Aclaraciones & Ejemplos \\
\hline $\begin{array}{c}\text { Describe la situación } \\
\text { de interés (0) }\end{array}$ & $\begin{array}{c}\text { No es reflexión en caso de que no salte } \\
\text { de un elemento a otro del hexágono. }\end{array}$ & $\begin{array}{c}\text { "No sabíamos que la guía estaba } \\
\text { incompleta" }\end{array}$ \\
\hline $\begin{array}{c}\text { Indica los motivos por } \\
\text { los que se ha dado una } \\
\text { situación (1) }\end{array}$ & $\begin{array}{c}\text { Supone la existencia de la descripción } \\
\text { previa de una situación, se considera un } \\
\text { nivel bajo de reflexión dentro de un } \\
\text { mismo elemento del hexágono. }\end{array}$ & $\begin{array}{c}\text { "Antes de hacer una actividad con los } \\
\text { estudiantes la tienes que hacer tu } \\
\text { primero como profesor para saber } \\
\text { cómo qué te puede pasar" }\end{array}$ \\
\hline $\begin{array}{c}\text { Explica las } \\
\text { consecuencias de } \\
\text { dicha situación (2) }\end{array}$ & $\begin{array}{c}\text { Supone la existencia de la descripción } \\
\text { previa de una situación, se considera un } \\
\text { nivel medio-bajo de reflexión dentro de } \\
\text { un mismo elemento del hexágono. }\end{array}$ & $\begin{array}{c}\text { "Había grupos que, aun conociendo } \\
\text { sobre botánica, se atascaban. } \\
\text { Imagínate alumnos que no han } \\
\text { trabajado en absoluto, se les hace } \\
\text { cuesta arriba" }\end{array}$ \\
\hline $\begin{array}{c}\text { Supone al menos la existencia de una } \\
\text { descripción previa de la situación. Puede } \\
\text { venir precedida de motivos o } \\
\text { Propone soluciones a } \\
\text { la situación (3) }\end{array}$ & $\begin{array}{c}\text { consecuencias de la misma. Implica, } \\
\text { para un mismo elemento un nivel de } \\
\text { reflexión medio. }\end{array}$ & $\begin{array}{c}\text { que no sabes, lo mejor es decir que } \\
\text { no lo sabes, si no los alumnos } \\
\text { cometen errores y se desconciertan } \\
\text { porque no les sale" }\end{array}$ \\
\hline
\end{tabular}

Fuente: elaboración propia, adaptado a partir de los niveles de reflexión de Hatton y Smith (1995)

Finalmente, para aunar las relaciones y el tipo de relación (si existiera) entre componentes en los distintos enunciados para cada tema de interés, se elaboró una figura hexagonal que representaba los diferentes elementos del hexágono del CDC en los que, dentro de cada uno de ellos, se diferencian niveles que van desde la descripción de la situación (0) hasta el planteamiento de soluciones (3), tal y como se muestra en el gráfico 2 . Sobre el hexágono se coloca la secuencia de enunciados (letras) en orden alfabético de acuerdo con su aparición temporal.

GRÁFICO 2. Esquema seguido en el análisis de las declaraciones y enunciados obtenidos. Los números de 0 a 3 representan el nivel reflexivo (véanse las tablas 1 y 2) y las letras (A, B, C...) representan el orden temporal de aparición de los componentes del enunciado

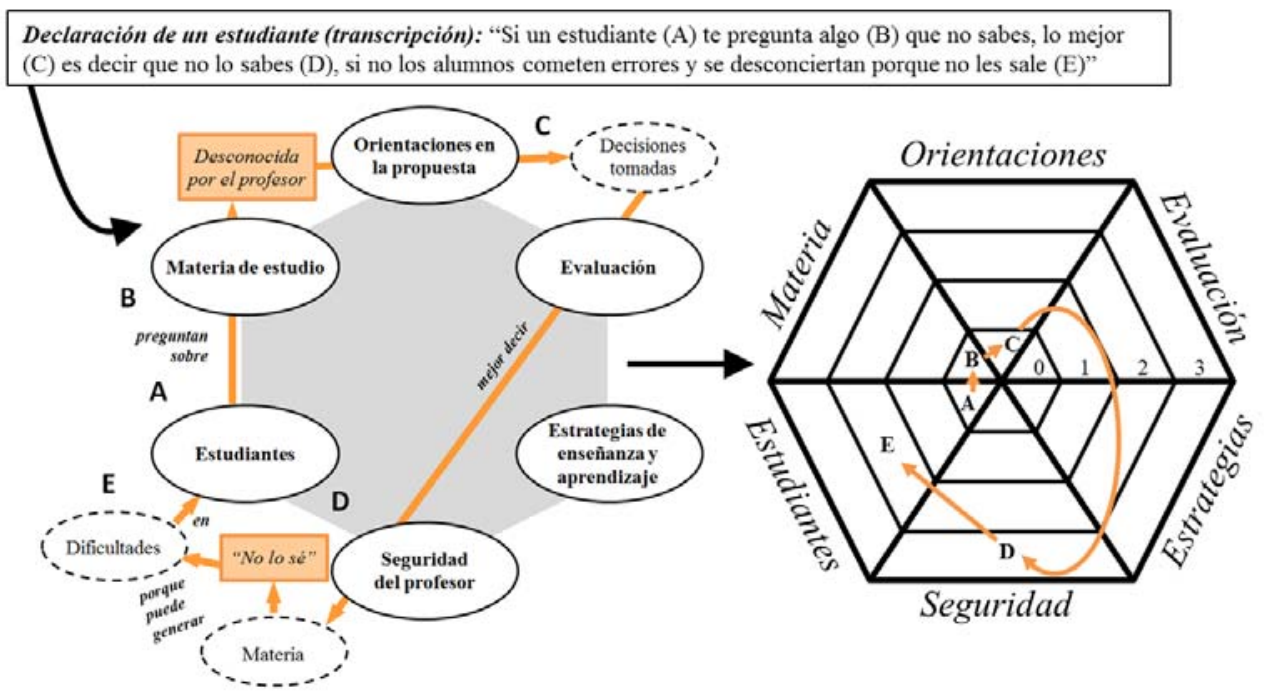

Fuente: elaboración propia 


\section{RESULTADOS}

En la sesión de discusión sobre la actividad realizada con los estudiantes descritos surgieron tres temas de interés: 1) lo que aprendieron durante la actividad, 2) la actuación ideal del profesor en el contexto de la misma y 3) cuál de los tres enfoques prácticos propuestos (relacionado con el orden de uso de los recursos) era el más idóneo. La sesión comenzó, no obstante, con una cuestión que generó bastante incertidumbre en los estudiantes: ¿cuál era el objetivo de la actividad realizada? Lo que llevó a un tipo de respuestas derivadas de la misma: lo que pensaban que habían aprendido durante la realización de la propuesta.

Tal y como se observa en el gráfico 3, a excepción del componente "seguridad del profesor", se pusieron en juego el resto de los componentes del hexágono del CDC (representados como elipses con borde negro y línea continua). A su vez, se pudieron concretar dentro de estos componentes algunos sub-componentes (elipses con borde negro y línea discontinua). A modo de ejemplo, mostramos uno de los enunciados para facilitar la interpretación del gráfico 3 .

GRÁFICO 3. Enunciados derivados de las declaraciones de los estudiantes para el tema de interés 1.

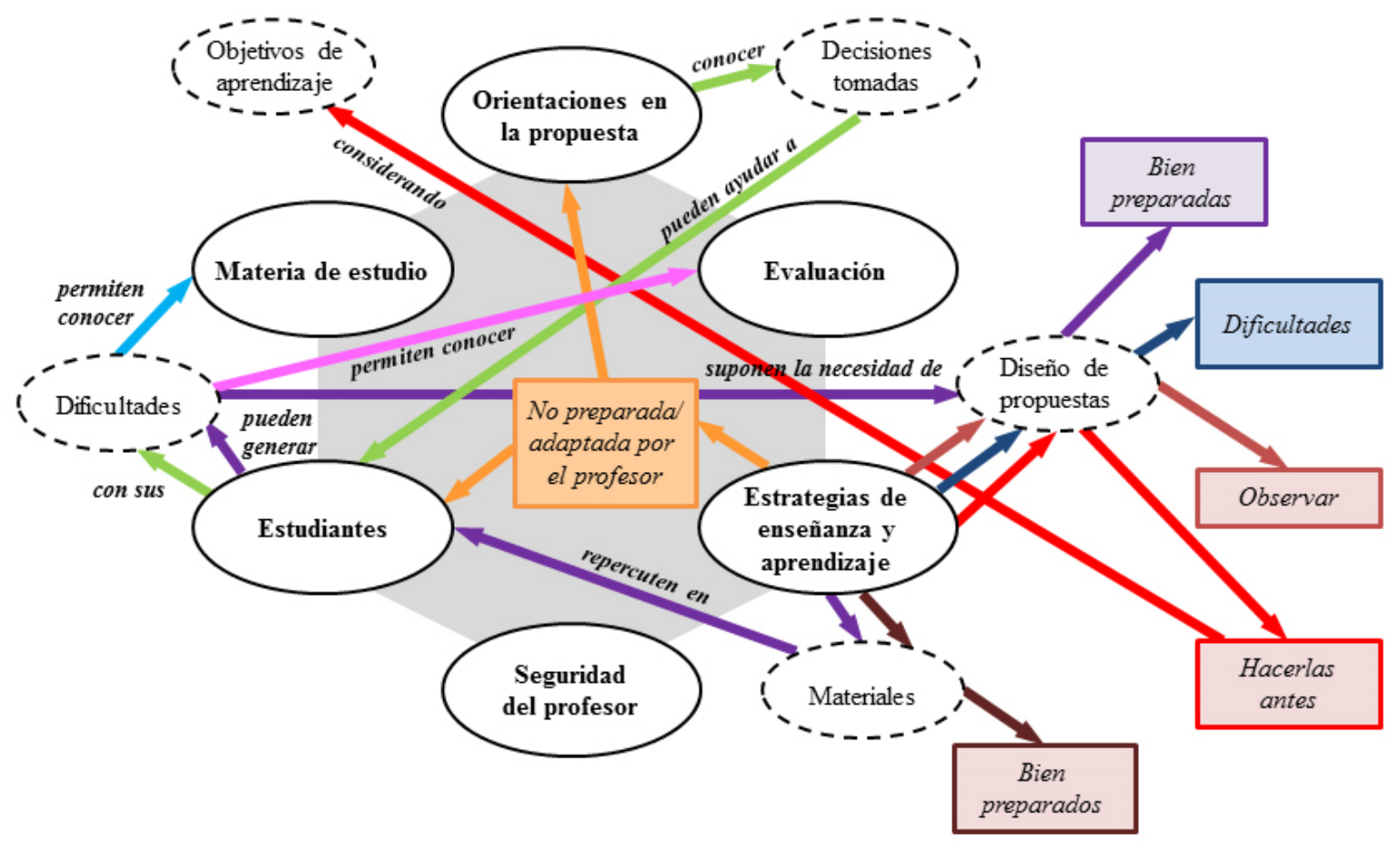

Fuente: elaboración propia

Cada enunciado comienza en un componente o sub-componente del hexágono y tiene la misma tonalidad de color. En recuadros en esa misma tonalidad aparecen palabras que ayudan a comprender dicho enunciado y sobre las flechas, que van orientando la dirección del mismo, aparecen nexos orientativos. Así, el enunciado marcado en color azul se leería del siguiente modo: "Saber sobre las dificultades con las que se encuentran los estudiantes nos permite conocer sobre la materia" o el marcado en marrón oscuro "Los materiales tienen que estar bien preparados". Globalmente son muchos los enunciados que se asocian al conocimiento de estrategias de enseñanza y aprendizaje. Otros, como los relacionados con el elemento "orientaciones en la propuesta" parten de las declaraciones realizadas por los estudiantes que ejercieron el papel de "profesores". 
El siguiente tema de interés surgió por iniciativa de uno de los estudiantes que había ejercido el rol de profesor (o experto) a lo largo de la secuencia (gráfico 4). Así, en uno de los enunciados se interpreta la necesidad, por parte del docente, de tener seguridad cuando los estudiantes plantean cuestiones sobre el tema que son desconocidos por el profesor (en naranja). Otros sin embargo hablan del rol del profesor desde una perspectiva "teórica" y de ahí que se incluya en el elemento estrategias de enseñanza y aprendizaje (en azul).

GRÁFICO 4. Enunciados derivados de las declaraciones de los estudiantes acerca del tema de interés 2.

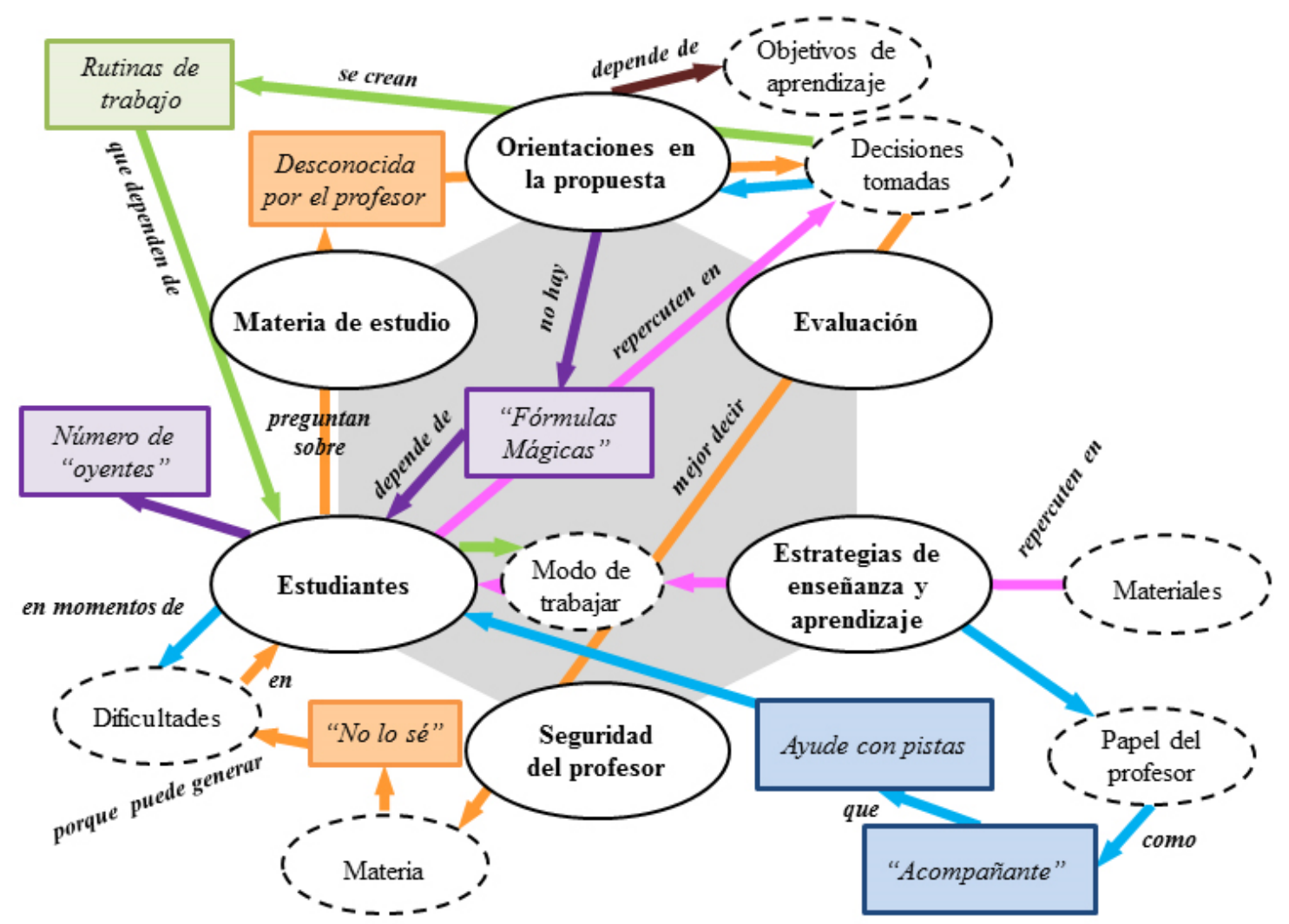

Fuente: elaboración propia

El último tema de interés (gráfico 5) sacó a la luz las creencias acerca de los objetivos de este tipo de propuestas. Como ejemplo, mientras que una declaración abogaba por trabajar previamente los conceptos desde una perspectiva aparentemente expositiva (en verde), otras parecían no tenerlo tan claro. Así, una de éstas trata de explicar los motivos por los cuales realizar la actividad práctica y sus consecuencias (en naranja). 
GRÁFICO 5. Enunciados derivados de las declaraciones de los estudiantes acerca del tema de interés 3.

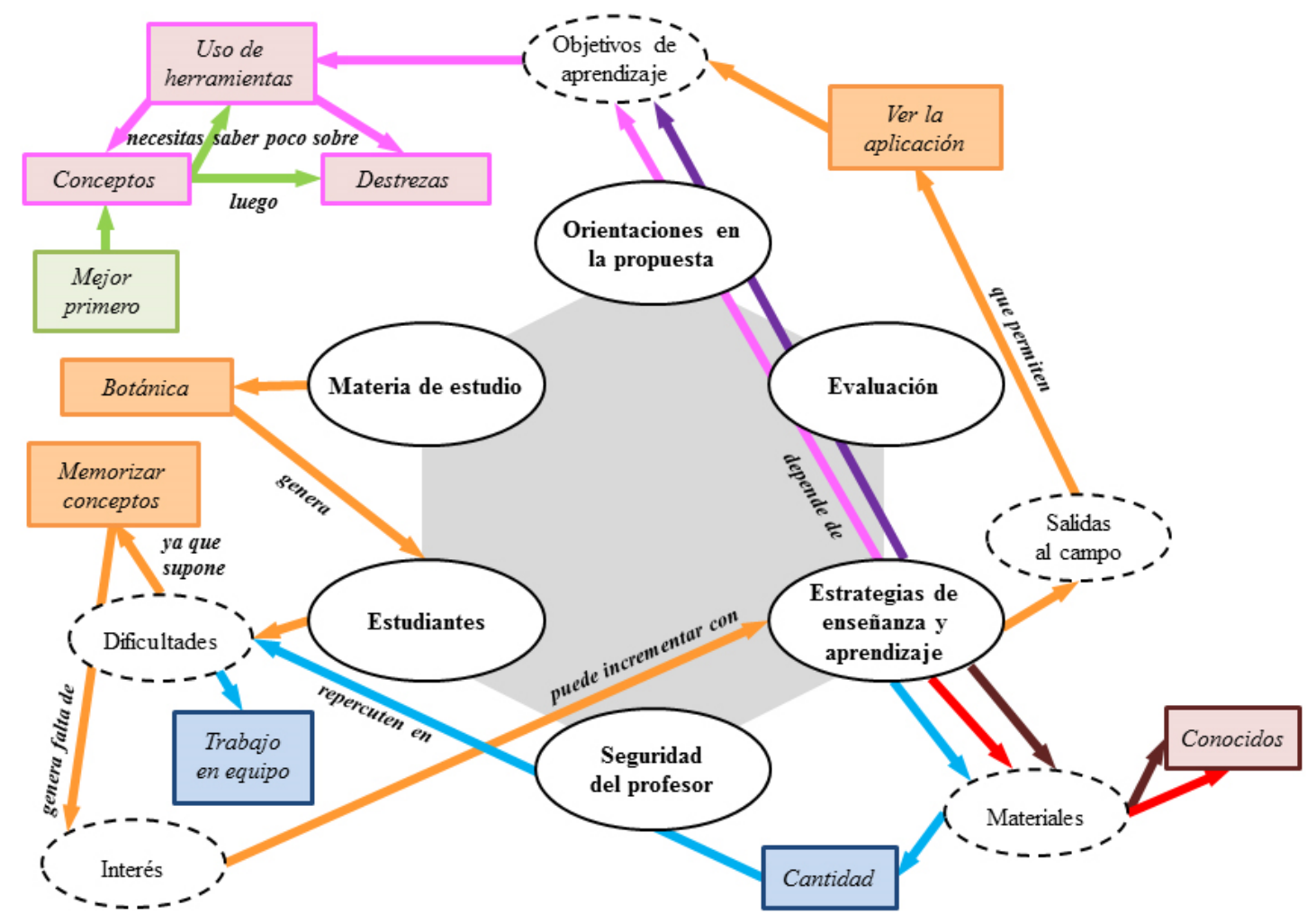

Fuente: elaboración propia

A nivel global, las figuras presentadas permiten resumir gráficamente los temas que generaron debate entre los estudiantes, el tipo de declaraciones realizadas, los componentes que tenían en consideración y las relaciones existentes entre ellos. Estas relaciones fueron sintetizadas en forma de diagramas hexagonales tal y como se muestra en los gráficos 6 y 7 .

A partir del discurso elaborado en el aula se han extraído 22 unidades de análisis que correspondían a enunciados que hacían referencia a uno o varios componentes del hexágono del CDC. De esta forma, se encuentran hasta 47 referencias a alguno de los componentes teniendo en cuenta que un mismo enunciado puede enmarcarse en un componente o presentar elementos de varios de ellos. La proporción de ocasiones en que se considera cada componente del CDC permite hacernos una idea de los principales aspectos sobre los que discutió el grupo de estudiantes. El gráfico 6 muestra los porcentajes de aparición de los enunciados a lo largo de la sesión. En él se observa una mayor aparición de enunciados relacionados con el conocimiento de los estudiantes, las estrategias de enseñanza y las orientaciones en la propuesta, apareciendo escasos enunciados relacionados con la materia, la seguridad del profesorado o la evaluación.

En detalle, entre los 22 enunciados analizados, se observa la presencia de 7 que se limitan a un único componente (enunciados no relacionales o estancos, en delante, EE) y 15 enunciados que relacionan varios componentes del hexágono (enunciados relacionales, en adelante, ER). Los EE tienen en todos los casos un carácter meramente descriptivo, apareciendo cinco en "Estrategias de enseñanza y aprendizaje" y dos en "Orientaciones en la propuesta". En cuanto a los ER, para visibilizar mejor las relaciones entre elementos del hexágono y el tipo de relaciones establecidas, optamos por sintetizar los gráficos 3,4 y 5 siguiendo la estrategia mostrada en el gráfico 2 . De esta manera, en el gráfico 7 se muestra la síntesis de resultados para cada tema de interés (gráfícos 7a, b y c) así como el recuento total de los elementos que conforman los ER (gráfico 7d). 
GRÁFICO 6. Porcentaje de aparición de los componentes del hexágono en los enunciados

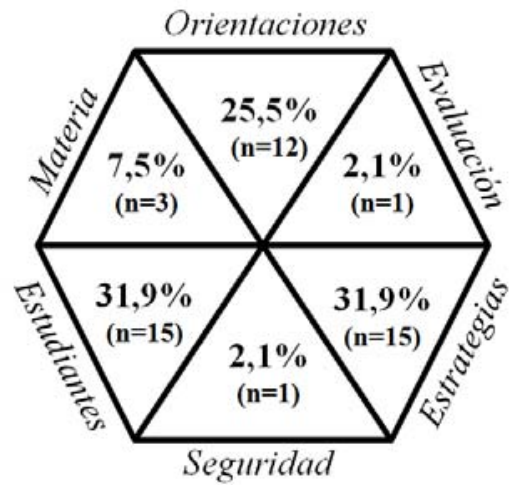

Fuente: elaboración propia

GRÁFICO 7. Tipos de relaciones entre componentes del CDC para cada tema de interés. Colores mostrados en figuras (a), (b) y (c) asociados a los enunciados de los gráficos 3, 4 y 5.

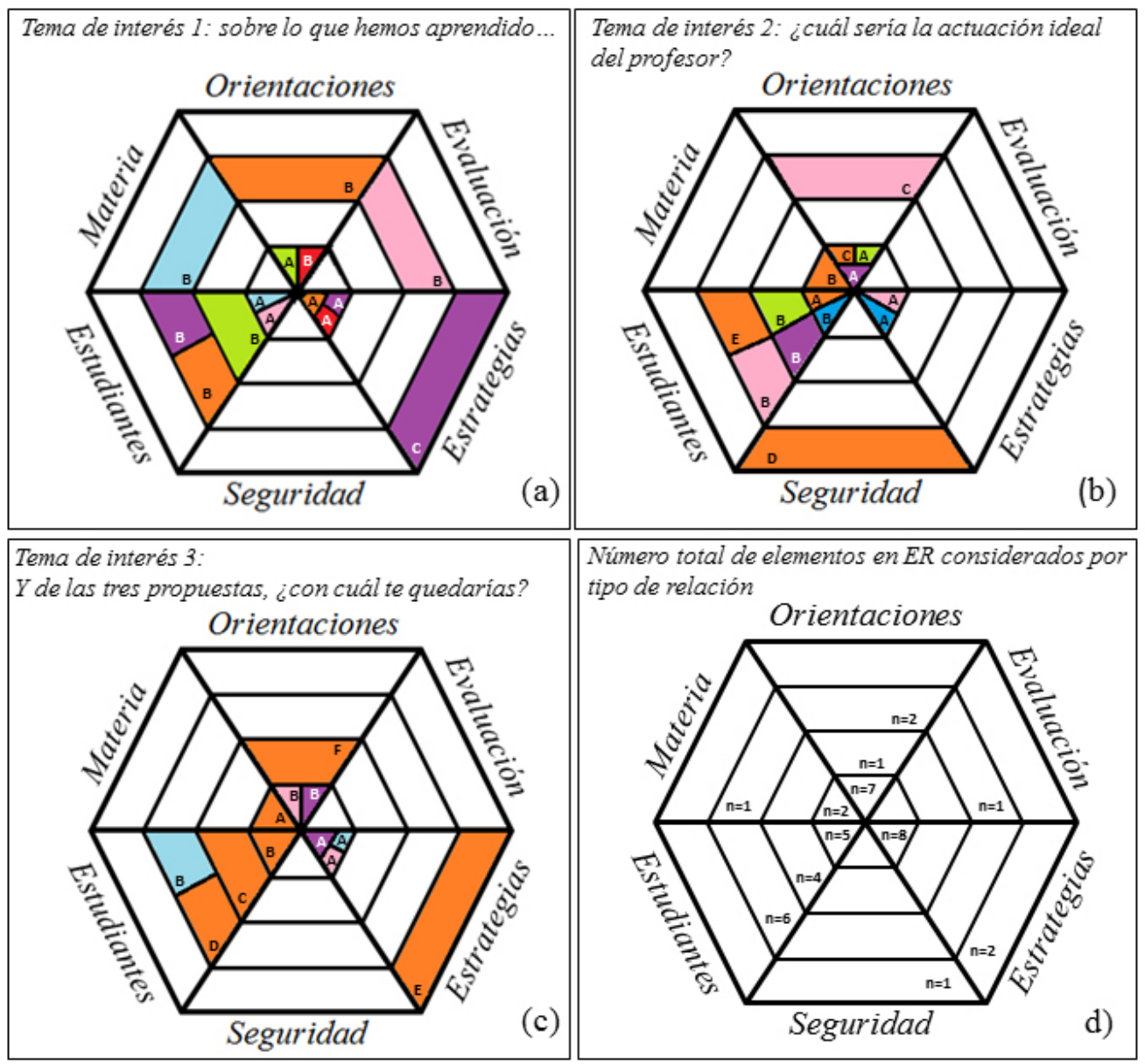

Fuente: elaboración propia

Para el tema de interés 1, tal y como se muestra en el gráfico 7a, se observan seis ER identificados con los mismos colores que aparecen para estos enunciados en el gráfíco 3. Así, del interior al exterior del hexágono aparecen los diferentes niveles de reflexión (niveles 0 a 3 de la tabla 2) a través de los cuales se relacionan los diferentes enunciados. Todos ellos comienzan con una descripción y van saltando a otros elementos del hexágono del mismo u otro nivel. De esta 
forma, en el siguiente componente se marca el tipo de relación que se ha establecido con el anterior, determinado por un nivel. Para seguir el proceso del ER, estos pasos se han organizado cronológicamente siguiendo una secuencia alfabética (A, B, C...). En caso de que el discurso tenga una bifurcación, se ha identificado con la misma letra el componente al que llega con la coloración correspondiente. Por ejemplo, el ER que más elementos abarca para el tema de interés 1 sufre una bifurcación (color naranja). Parte de una descripción relacionada con las estrategias de enseñanza y aprendizaje y explica las consecuencias que supone en los estudiantes dicha estrategia (componente estudiantes, nivel 2) y el modo en que el profesor orienta la propuesta (componente orientaciones, nivel 2).

Sobre este tipo de enunciados llama la atención que aquellos que proponen soluciones pasan previamente por una valoración de las consecuencias o motivos de la situación. Asimismo, dos de ellos, explican las consecuencias de las soluciones dadas o dan motivos por los que llevarlas a cabo. Considerando esta clasificación, en este contexto nos encontramos con diferentes enunciados que pueden permitir hacernos una idea del nivel de reflexión sobre la experiencia llevada a cabo. En la tabla 3 mostramos los porcentajes de cada tipo de enunciado.

Se observa que cinco de los enunciados relacionan más de un componente del CDC (niveles 2 y 3), alcanzando el nivel 3 tan solo tres enunciados de los 22 analizados. Por ejemplo, en el tema de interés 3 (en naranja en el gráfico 7c) se relaciona la temática abordada (botánica) con la dificultad que genera a los estudiantes (aprender muchos nombres, por ejemplo) y que, como consecuencia, genera una falta de interés en éstos. Como solución se propone abordar este tipo de actividades desde un enfoque aplicado (uso de las salidas de campo, conocer las características a partir de la elaboración de claves dicotómicas), aclarando que como profesores uno de los objetivos debería ser que los estudiantes de secundaria viesen el sentido práctico de la temática abordada. En el punto opuesto aparecen siete declaraciones que se centran en un único componente del CDC.

TABLA 3. Frecuencia de aparición de los diferentes tipos de enunciados

\begin{tabular}{cccccc}
\hline Tipo de Enunciado & $\begin{array}{c}\text { Estanco } \\
(\mathrm{EE}, \mathrm{n}=7)\end{array}$ & \multicolumn{3}{c}{$\begin{array}{c}\text { Relacional } \\
(\mathrm{ER}, \mathrm{n}=15)\end{array}$} \\
\hline $\begin{array}{c}\text { Número de nexos entre } \\
\text { componentes }\end{array}$ & Ninguno & \multicolumn{2}{c}{ Uno } & \multicolumn{2}{c}{ Más de uno } \\
\hline $\begin{array}{c}\text { Tipo de relación ente } \\
\text { niveles }\end{array}$ & -- & Nivel 0 & Nivel 1 ó 2 & Nivel 2 & Nivel 3 \\
\hline $\begin{array}{c}\text { Frecuencia respecto al } \\
\text { total de enunciados }\end{array}$ & $7 / 22$ & $4 / 22$ & $6 / 22$ & $2 / 22$ & 3 \\
\hline
\end{tabular}

\section{DisCUSión DE LOS RESUlTAdOS}

La discusión con el grupo-clase ha permitido conocer el tipo de componentes del Conocimiento Didáctico del Contenido que manejan los estudiantes durante la actividad. Se observa que en el caso analizado, estos estudiantes apenas consideran los componentes seguridad y evaluación y, por el contrario, su discurso se centra mayoritariamente en el conocimiento de los estudiantes de secundaria y en las estrategias didácticas (gráfico 5). Existe una coherencia entre la proporción de los componentes mencionados y los aspectos trabajados en la propuesta reflexiva, ya que: 1) en los cuestionarios analizados (Sáez Bondía y Cortés Gracia, 2019) se hace énfasis en las posibles dificultades que podrían tener los estudiantes de secundaria; 2) en la propuesta se emplean diferentes estrategias para abordar una misma actividad; 3) la evaluación de los aprendizajes queda en un segundo plano y no se tiene en consideración explícitamente en la propuesta reflexiva y 4) la seguridad del profesor es mencionada únicamente por aquellos estudiantes que asumen ese papel 
durante la propia actividad. Esto nos lleva a pensar en la necesidad de hacer más explícitos los componentes menos considerados por los estudiantes cuando se plantean propuestas en la formación inicial del profesorado. Por ejemplo, introducir la evaluación como parte de la propuesta reflexiva con el objetivo de cambiar a modelos evaluativos más alejados de contextos tradicionales, tal y como proponen Delord y Porlán (2018).

Por otra parte, los estudiantes no solo consideran todos los componentes del hexágono del CDC (Park y Oliver, 2008), sino que establecen relaciones entre ellos en numerosas ocasiones. Esa relación saca a la luz el pensamiento reflexivo de los mismos sobre las situaciones vividas en la actividad propuesta (Nilsson 2008, Park y Oliver, 2008). Cuando los estudiantes exponen sus percepciones, bien retoman sus experiencias y las describen, bien se plantean motivos por los cuales han sucedido o bien se predicen sus consecuencias. Incluso son capaces de proponer soluciones más o menos fundamentadas y predecir las posibles consecuencias de las mismas. Así pues, se podría decir que la secuencia de actividades llevadas a cabo favorece, hasta cierto punto, el desarrollo de una reflexión sobre la acción (Schön, 1998). La sesión de discusión parece favorecer que los profesores en formación inicial se muestren más reflexivos (Goodman, 1987), ya que permite poner sobre la mesa problemas sustantivos relacionados con la enseñanza y el aprendizaje en el contexto de las salidas de campo. No obstante, para poder validar esta afirmación es necesario profundizar en los cambios en las ideas de los estudiantes a lo largo de la secuencia planteada y cómo estas se utilizan en la sesión de discusión analizada (Sáez Bondía y Cortés Gracia, 2019).

La estrategia de análisis mostrada en este trabajo nos permite conocer las ideas que tienen los estudiantes sobre la propuesta vivida y el nivel de reflexión que alcanzan dichas ideas. El uso de la categorización planteada por Hatton y Smith (1998) dejaba de lado algo fundamental: el saber del profesor. El uso del CDC (Park y Oliver, 2008) nos ha permitido enmarcar la reflexión bajo unos componentes didácticos relevantes en la formación del profesorado. La reflexión parece no ser ajena al Conocimiento Didáctico del Contenido de una disciplina y tal vez este sea uno de los problemas con el que diferentes autores se han encontrado a la hora de tratar de evaluarla.

$\mathrm{Al}$ aunar estos dos enfoques se observa que los niveles de reflexión previamente establecidos por Hatton y Smith (1995) sufren alguna modificación en el contexto analizado. Podríamos incluir hasta cuatro niveles reflexivos teniendo en cuenta el discurso. Siendo 0 la ausencia de reflexión y 4 un nivel medio-alto, tal y como se muestra en la tabla 4.

Esta tabla no solo ha considerado las relaciones obtenidas en el análisis sino aquellas que se podrían establecer y que no han surgido en el contexto estudiado. Así, en las declaraciones de los estudiantes y atendiendo a esta nueva categorización, tan solo tres de éstas alcanzan niveles medioaltos de reflexión sobre la situación analizada. Los niveles altos requerirían una mayor profundización en la propuesta de soluciones, si bien en este caso, aunque se proponen algunas, son planteadas de forma bastante superficial. Esta categorización de niveles parece adecuada para este caso concreto, aunque ésta podría adaptarse a cada contexto, aspecto que abre nuevas líneas de investigación en torno a la evaluación de la reflexión desde la perspectiva del CDC.

La reflexión sobre situaciones de aula es compleja y requiere de conocimientos sobre distintos aspectos de la enseñanza: sobre la materia, sobre las visiones de los estudiantes, sobre las diferentes estrategias de enseñanza y aprendizaje, sobre las posibles orientaciones a dar en una propuesta y sobre la creación de una identidad profesional. Este trabajo ha mostrado la utilidad de una estrategia para evaluar la reflexión a partir de un ejemplo derivado del análisis del discurso que es transferible a cualquier otro contexto profesionalizante adaptando los elementos del CDC para ese contexto. Asimismo, hay que destacar que la explicitación de las ideas de los estudiantes en la sesión de puesta en común permite visibilizar con más claridad sus preocupaciones y la visión que tienen sobre la actividad realizada: "Por supuesto, hay procesos cognitivos, pero estos son propios de las prácticas discursivas que están justo enfrente de nuestras narices” (traducción de Harré, 1992, p.6). 
TABLA 4. Propuesta de categorización de niveles de reflexión en el discurso sobre una situación de aula en la formación del profesorado.

\begin{tabular}{|c|c|c|}
\hline $\begin{array}{l}\text { Niveles } \\
\text { reflexión }\end{array}$ & Características & Ejemplos \\
\hline Nivel 0 & $\begin{array}{l}\text { Se describen situaciones considerando un } \\
\text { solo componente del CDC. No es } \\
\text { reflexión. }\end{array}$ & $\begin{array}{l}\text { "Hemos aprendido a conocer las dificultades que } \\
\text { tiene el diseño de una salida de campo." }\end{array}$ \\
\hline Nivel 1 & $\begin{array}{l}\text { Se asocian una vez dos componentes del } \\
\text { CDC a nivel descriptivo o sobre un } \\
\text { mismo componente se describe la } \\
\text { situación y establecen relaciones causales } \\
\text { (se dan motivos o consecuencias de la } \\
\text { situación descrita). Nivel muy bajo. }\end{array}$ & $\begin{array}{l}\text { "Un buen profesor tiene que ser un acompañante que } \\
\text { en momentos de bloqueo del grupo (de estudiantes), } \\
\text { con pistas, consiga que avancen." }\end{array}$ \\
\hline Nivel 2 & $\begin{array}{l}\text { Se asocian una vez dos componentes del } \\
\text { CDC a través de relaciones causales o } \\
\text { bien, sobre un mismo componente se } \\
\text { llegan a proponer soluciones sobre la } \\
\text { situación precedida de relaciones } \\
\text { causales. Nivel bajo. }\end{array}$ & $\begin{array}{l}\text { "El papel de X (rol de profesor), que comenzó la } \\
\text { actividad con nosotros, partía con la misma } \\
\text { situación... (como estudiantes) nos habíamos } \\
\text { acostumbrado, habíamos cogido unas pautas sobre } \\
\text { cómo trabajar, sabía más por dónde ir y sin embargo } \\
\text { para otros grupos de estudiantes la forma de trabajar } \\
\text { de Y (rol de profesor) era mejor." }\end{array}$ \\
\hline Nivel 3 & $\begin{array}{l}\text { Se asocian dos o más componentes del } \\
\text { CDC más de una vez estableciéndose } \\
\text { entre ellos una relación causal. Nivel } \\
\text { medio }\end{array}$ & $\begin{array}{l}\text { "Hay que darse cuenta del material del que se } \\
\text { dispone. No puedes (como profesor) tener a seis } \\
\text { estudiantes con una guía porque solo se enteran dos } \\
\text { personas. El que lleva la guía y el que le apoya se } \\
\text { enteran y el resto son apuntadores. Eso afecta a cómo } \\
\text { llevar al grupo (como profesores)." }\end{array}$ \\
\hline Nivel 4 & $\begin{array}{l}\text { Se asocian dos o más componentes del } \\
\text { CDC más de una vez llegando a proponer } \\
\text { soluciones para la situación precedidos de } \\
\text { una relación causal. Nivel medio-alto. }\end{array}$ & $\begin{array}{l}\text { "Trabajar la botánica y luego ver la puesta en } \\
\text { práctica... es lo mejor. La botánica para los alumnos } \\
\text { es dura, aprender términos y palabras nuevas es un } \\
\text { esfuerzo de memoria para ellos y como no ven la } \\
\text { parte práctica (...). Utilizar la guía didáctica (de } \\
\text { identificación) para hacer una actividad fuera del } \\
\text { aula, supongo que es una forma de motivar, para que } \\
\text { ellos vean la aplicación práctica que pueden tener } \\
\text { unos conocimientos básicos de botánica, por } \\
\text { ejemplo, que conozcan que el pino tiene acículas", } \\
\text { porque si igual la forma de la hoja la han visto en } \\
\text { clase, no le ven la aplicación práctica." }\end{array}$ \\
\hline
\end{tabular}

Fuente: elaboración propia

\section{Referencias}

Acevedo, J.A. (2009). Conocimiento didáctico del contenido para la enseñanza de la naturaleza de la ciencia (I). Revista Eureka sobre Enseñanza y Divulgación de las Ciencias, 6(1), 21-46. DOI: 10.25267/Rev_Eureka_ensen_divulg_cienc.2009.v6.i2.01

Amobi, F.A. (2005). Preservice teachers' reflectivity on the sequence and consequences of teaching action in a microteaching experience. Teacher Education Quarterly, 32(1), 115-130.

ANECA (2005). Libro blanco. Título de Grado en Magisterio. Volumen 1. Recuperado de http://www.aneca.es/var/media/150404/libroblanco jun05 magisterio1.pdf [21 de junio de 2017]. 
Borowski, A., Carlson, J., Fischer, H.E., Henze, I., Gess-Newsome, J., Kirschner, S. y van Driel, J. H. (2011). Different models and methods to measure teachers' pedagogical content knowledge. Comunicación presentada en ESERA 2011 Conference: Science learning and Citizenship, Lyon, Francia.

Carrasquer, J., Álvarez, M.V., Lafuente, A. y Pérez, I. (2001). Nuestros amigos los árboles y

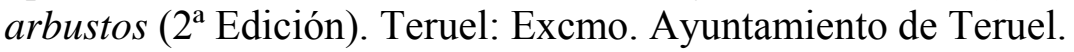

Delord, G. y Porlán, R. (2018). Del discurso tradicional al modelo innovador en enseñanza de las ciencias: obstáculos para el cambio. Didáctica de las ciencias experimentales y sociales, 35, 77-90. DOI: 10.7203/DCES.35.12193

Dewey, J. (1938). Logic: The Theory of Inquiry. Nueva York: William Morrow.

Dewey, J. (1989). Cómo pensamos. Nueva exposición de la relación entre pensamiento reflexivo y proceso educativo. Barcelona: Paidós.

Eraut, M. (1994). Developing professional knowledge and competence. Londres: Falmer Press.

Fernández-Fernández, S., Arias-Blanco, J.M., Fernández-Alonso, R., Burguera-Condon, J. y Fernández-Raigoso, M. (2016). Pensamiento reflexivo e investigador en educación. Aspectos a tener en cuenta en la formación del profesorado. Revista ELectrónica de Investigación y EValuación Educativa, 22, 1-17. DOI: 10.7203/relieve.22.2.8425

Gaudin, C. y Chaliès, S. (2015). Video viewing in teacher education and professional development: A literature review. Educational Research Review, 16, 41-67. DOI: 10.1016/j.edurev.2015.06.001

Goodman, J. (1987). Reflexión y formación del profesorado. Estudios de casos y análisis teórico. Revista de educación, 234, 223-244.

Harré, R. (1992). The Second Cognitive Revolution. American Behavioral Scientist, 36(1), 5-7.

Hatton, N. y Smith, D. (1995). Reflection in teacher education: Towards definitions and implementation. Teaching and Teacher Education, 11, 33-49. DOI: 10.1016/0742051X(94)00012-U

Imbernón, F. (2017). Ser docente en una sociedad compleja. La difícil tarea de enseñar. Barcelona: Graó.

Lledó, A. y Giner, A. (2009). Un proyecto colaborativo en la facultad de Educación. Guías docentes de la titulación de maestro. Alicante: Marfil / Universidad de Alicante.

Loughran, J.J. (2002). Effective Reflective Practice: In Search of Meaning in Learning about Teaching. Journal of Teacher Education, 53(1), 33-43. DOI: 10.1177/0022487102053001004

Lussi Borer, V., Ria, L., Durand, M. y Muller, A. (2014). How Do Teachers Appropriate Learning Objects Through Critical Experiences? A Study of a Pilot In-School Collaborative Video Learning Lab. Open Journal per La Formazione in Rete, 14(1), 63-74. DOI: $10.13128 /$ formare-15137

Marcelo, C. (1993). Cómo conocen los profesores la materia que enseñan. Algunas contribuciones de la investigación sobre Conocimiento Didáctico del Contenido. En L. Montero y J.M. Vez (Eds.), Las didácticas específicas en la formación del profesorado (pp. 151-186). Santiago de Compostela: Tórculo.

Medina, J. L. y Jarauta, B. (2013). Análisis del conocimiento didáctico del contenido de tres profesores universitarios. Revista de educación, 360, 600-623. DOI: 10.4438/1988-592X-RE2011-360-131

Mena, M., García Rodríguez, M.L. y Tillema, H. (2012). Student teacher reflective writing: What does it reveal? European Journal of Teacher Education, 1, 1-17. DOI: $10.1080 / 02619768.2012 .713933$

Nilsson, P. (2008). Teaching for understanding: The complex nature of pedagogical content knowledge in pre-service education. International Journal of Science Education, 30 (10), 1281-1299. DOI: 10.1080/09500690802186993 
Nóvoa, A. (2009). Para una formación de profesores construida dentro de la profesión. Revista de Educación, 350, 203-218.

Orion, N. y Hofstein, A. (1994). Factors that influence learning during a scientific field trip in a natural environment. Journal of Research in Science Teaching, 31(10), 1097-1119.

Park, S. y Oliver, J.S. (2008). Revisiting the Conceptualisation of Pedagogical Content Knowledge (PCK): PCK as a conceptual Tool to Understanding Teachers as Professionals. Research on Science Education, 38, 261-284. DOI: 10.1007/s11165-007-9049-6

Parker, J. (1992). Discourse dynamics. Londres: Routledge.

Pedrinaci, E. (2012). Trabajo de campo y aprendizaje de las ciencias. Alambique. Didáctica de las Ciencias Experimentales, 71, 81-89.

Peinado, M. y Abril, A.M. (2016). El Máster en Profesorado de Secundaria desde dentro: expectativas y realidades del Prácticum. Didáctica de las Ciencias Experimentales y Sociales, 30, 5-22. DOI: 10.7203/DCES.30.6811

Pérez Gómez, A.I. (2010). El sentido del prácticum en la formación de docentes. La compleja interacción de la práctica y la teoría. En A.I. Pérez Gómez (coord.), Aprender a enseñar en la práctica: procesos de innovación y prácticas de formación en la Educación Secundaria (pp. 89-106). Barcelona: Graó / MEC

Perrenoud, P. (2004). Desarrollar la práctica reflexiva en el oficio de enseñar. Barcelona: Graó.

Ravanal Moreno, E. y López-Cortés, F. (2016). Mapa del conocimiento didáctico y modelo didáctico en profesionales del área biológica sobre el contenido de célula. Revista Eureka sobre Enseñanza y Divulgación de las Ciencias, 13(3), 725-742. DOI: 10.25267/Rev_Eureka_ensen_divulg_cienc.2016.v13.i3.15

Rich, P.J. y Hannafin, M. (2009). Technologies to Scaffold, Structure, and transform Teacher Reflection. Journal of Teacher Education, 60(1), 52-67. DOI: 10.1177/0022487108328486

Rodgers, C. (2002). Defining reflection: Another Look at John Dewey and Reflective Thinking. Teacher College Record, 104(4), 842-866.

Rusell, T. (2012). Cambios paradigmáticos en la formación del profesorado: peligros, trampas y promesas no cumplidas. Encounters on Education, 13, 71-91. DOI: 10.15572/ENCO2012.05

Sáez Bondía, M.J. y Cortés Gracia, A.L. (2019) ¿Cómo cambian las ideas de los estudiantes de Máster de Profesorado sobre una actividad práctica de campo tras su vídeo-análisis y discusión en pequeños grupos? Revista Eureka sobre Enseñanza y Divulgación de las Ciencias, 16(2), 2602. DOI: 10.25267/Rev_Eureka_ensen_divulg_cienc.2019.v16.i2.2602

Schön, D. (1992). La formación de profesionales reflexivos. Hacia un nuevo diseño de la enseñanza y el aprendizaje en las profesiones. Barcelona: Paidós.

Schön, D. (1998). El profesional reflexivo. Cómo piensan los profesionales cuando actúan. Barcelona: Paidós.

Shulman, L.S. (1986). Those who understand: knowledge growth in teaching. Educational Researcher, 15(2), 4-14.

Shulman, L.S. (1987). Knowledge and teaching: foundations of the new reform. Harvard Educational Review, 57(1), 1-22.

Simon, S. y Campbell, S. (2012). Teacher learning and professional development in science education. En B. Fraser, K. Tobin y McRobbie C.J. (Eds.) Second international handbook of science education (pp. 307-321). Springer Netherlands.

Simons, H. (2011). El estudio de caso: teoría y práctica. Madrid: Morata.

Smith, D. (1991). Educating the reflective practitioner in curriculum. Curriculum, 12, 115-124.

Toom, A., Husu, J. y Patrikainen, S. (2014). Student teachers' patterns of reflection in the context of teaching practice. European Journal of Teacher Education, 38(3), 1-21. DOI: 10.1080/02619768.2014.943731

Van Manen, M. (1977). Linking ways of knowing within ways of being practical. Curriculum Inquiry, 6, 205-228 
Yoon, H.G. y Kim, M. (2010). Collaborative reflection through dilemma cases of science practical work during practicum. International Journal of Science Education, 32(3), 283-301. DOI: 10.1080/09500690802516538

Zeichner, K.M. (1987). Preparing reflective teachers: An overview of instructional strategies which have been employed in preservice teacher education. International Journal of Educational Research, 11(5), 565-575.

\section{CÓMO CITAR ESTE ARTÍ́CULO}

Sáez Bondía, M. J. y Cortés García, A. L. (2019). ¿Cómo evaluar la reflexión sobre la práctica docente? Un ejemplo en la formación inicial del profesorado de Biología y Geología. Didáctica de las ciencias experimentales y sociales, 37, 127-146. DOI: 10.7203/DCES.37.14797 
